\title{
A Preliminary Kinetic Analysis for First Order Gas-Solid Reactions with Distributed Reaction Rate Constants Using Laplace Transform
}

\author{
S. P. MEHROTRA ${ }^{1)}$ and A. C. D. CHAKLADER ${ }^{2)}$ \\ 1) Department of Metallurgical Engineering, Indian Institute of Technology, Kanpur-208016, India. \\ Engineering, University of British Columbia, Vancouver, B.C., Canada V6T 1 W5.
}

2) Department of Metallurgical

(Received on January 25, 1988; accepted in the final form on September 9, 1988)

\begin{abstract}
A new approach has been developed to analyse the kinetics of heterogeneous gas-solid reactions. The solid reactant is considered as consisting of reactant species having different overall reaction rate constants and thus a rate constant distribution is associated with the solid. This distribution arises due to chemical heterogeneity of the material, availability of varying amounts of gaseous reactants at various locations within the particle due to its microstructural effects, and other physical phenomena which offer resistance to the reaction. Appropriate mathematics has been developed to express the reaction kinetics in terms of this rate constant distribution. The problem of evaluating the distribution is recognized to be equivalent to the problem of finding the inverse of the Laplace transform. A numerical method, based on non-linear optimization, has been used to carry out inversion of the Laplace transform and the rate constant distribution evaluated employing specified kinetic data. The applications of this new concept are illustrated using the kinetic data for reduction of hematite with hydrogen, and gasification of carbon with carbon dioxide. Effects of some of the operating parameters, e.g., temperature, particle size, porosity, gas flow rate and gas composition on the reaction kinetics are discussed in terms of this new concept.
\end{abstract}

KEY WORDS: reaction kinetics; gas-solid reactions; distributed rate constant; hematite reduction; carbon gasification.

\section{Introduction}

The conventional method of analysing the reaction kinetics of gas-solid reactions requires identification of various kinetic steps involved in the reaction, and evaluation of their rates. The kinetic steps which are generally considered include ${ }^{1)}$ : 1) diffusion of gaseous reactant from the bulk gas to the particle surface, 2) diffusion of the reactant through the product layer to the reaction interface, 3 ) adsorption of the gaseous reacting species at the interface, 4) reaction at the interface, 5 ) diffusion of the gaseous product away from the interface, 6) diffusion of the gaseous product through the product layer to the particle surface, and 7) transport of the product gas into the bulk gas stream. One of the commonly used techniques of identifying the reaction mechanism is to plot experimentally estimated conversion as a function of time. Usually a straight-line relationship between the two according to a certain type of equation is taken as evidence for the mechanism associated with the particular expression. However, more often straight lines with similar degrees of scatter are obtained for experimental data when they are plotted according to two different mechanisms. It has been conclusively shown by several investigators that certain straight-line relationships between variables over a limited range can not be regarded as evidence for control by a given proposed mechanism. ${ }^{2-5)}$ When diffusion and chemical reactions are of comparable importance, the task of indentifying reaction mechanism becomes cven more difficult.
Attempts have been made to develop generalized models to include the contributions of both chemical reaction and diffusion controls to the overall reaction rate. ${ }^{4-9)}$ However, these too have found only limited applications. These models have difficulty in the application for a system of changing structure. Further, the uncertainties in the values of parameters (like the diffusion cocfficient, tortuosity factor, etc.) involved in these computations make the predictions unreliable.

In view of the difficulty and the uncertainties involved in identification of correct reaction mechanism, which may even undergo change during the course of reaction, it is worth exploring the possibility of analysing kinetic data using an approach which is independent of reaction mechanism. In what follows in this paper is an approach that has been developed to analyse the kinetics of first order gas-solid reaction using the concept of distributed reaction rate constant. The rate constant used in the algorithm is a lumped parameter which represents the combined effects of physical and chemical characteristics of the reacting species, operating experimental conditions as well as the contribution of all kinetic steps.

\section{Concept of Distributed Reaction Rate Con- stant}

For a truly chemical reaction controlled first order gas-solid reaction the rate can be expressed as

$$
\frac{d W(t)}{d t}=-k W(t)
$$


where $W(t)$ is the mass of the solid reactant at any given time $t$ and $k$ is the reaction rate constant. Such an cquation is also valid for those heterogeneous gas solid reactions where both chemical reaction and mass transfer process contribute to the overall rate but the internal pore structure does not change during the course of the reaction. 8,10 )

If $W_{0}$ is the initial mass of the reactant, Eq. (1) in its integrated form will be

$$
W(t)=W_{0} \exp [-k t]
$$

Thus, a semilogarithmic plot of fraction of unreacted solid $v s$. time would be a straight line with slope equal to $-k$. In most cases, however, there is deviation from the straight-line behaviour. One such plot for the experimental data taken from Ref. 11) is shown in Fig. 1. This deviation is often attributed to the resistance offered to the reaction by various physical phenomena.

As stated above, the overall reaction rate depends not only on the rate of the chemical reaction between the reactant (which is only one of the kinetic steps) but also on various physical factors the contribution of which to the overall rate is a function of time. For example, when a particle (a particle here would mean a single particle or a compact of particles or static bed of particles) is subjected to chemical reaction with a reactant gas, depending on reaction temperature, gas flow rate, chemical as well as physical properties (including internal microstructure) of reactant solid, the reaction may first begin at the external surface of the solid which is exposed to the reactant gas. With the progress of the reaction as the solid reactant at the external surface begins to deplete the effect of diffusion rate into the pores becomes more and more predominant. Until unless the porosity of the particle is high enough the reactant gas would be able to reach the inner most surface only towards the end of the reaction. In a given solid, the various fractions of solid mass contained in the particle are, therefore, exposed to the reactant gas enabling them to chemically react only at different

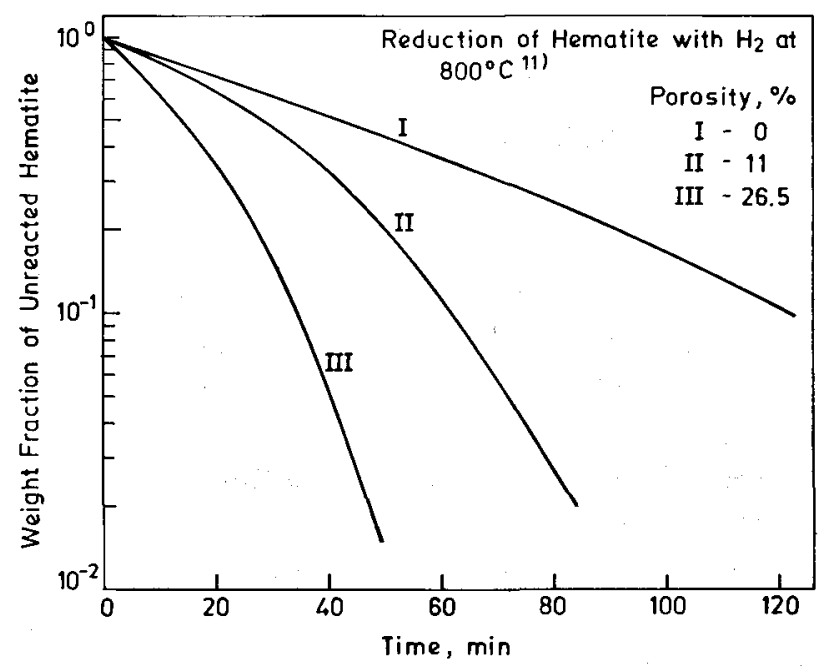

Fig. 1. Weight fraction of unreacted hematite as a function of time. stages of time. Thus, even if these various fractions of solid mass have the same intrinsic chemical reactivity, the probability of their reacting with the reactant gas at a given instant of time would be different. In other words, one may visualize, at least conceptually, the solid reactant as consisting of various reactant species which have different overall (apparent) reaction rate constants. Such an approach would also take into consideration the inherent heterogeneities in intrinsic chemical properties of various mass fractions contained in the solid. Hence, one may associate a rate constant distribution with the reactant solid. Thus, this distribution could arise due to chemical heterogeneities, availability of varying amounts of gaseous reactant at various locations within the particle due to its inner microstructure, and other physical phenomena which offer resistance to the reaction. Truly speaking this concept of distributed rate constant is not new to reaction kinetics. It has been used in chemical engineering literature to describe continuum of reactions ${ }^{12)}$ and for mixtures with parallel first order irreversible reactions. ${ }^{13)}$ In mineral engineering it has been used fairly extensively to describe the kinetics of some of the well-known physical separation processes, e.g., froth flotation. ${ }^{14,15)}$

If the initial mass of the solid reactant is normalized to unit mass and $\phi\left(k_{i}, 0\right)$ is the fraction of solid at time $t=0$ having overall reaction rate constant $k_{i}$, then

$$
W_{0}=\sum_{i=1}^{N} \phi\left(k_{i}, 0\right)=1
$$

where $\mathcal{N}$ is the number of fractions of solid having different rate constants. Henceforth, these fractions will be referred to as 'species'. Thus, $\phi\left(k_{i}, 0\right) ; i=$ $1, \ldots, \mathcal{N}$ represents a discrete rate constant distribution of $\mathcal{N}$ species.

\section{Formulation of Kinetics Problem}

If it is assumed that each of $\mathcal{N}$ species contained in the solid reacts with the reactant gas independently of each other, and that the reaction kinetics follows first order law, the amount of species $i$ (having reaction rate constant $k_{i}$ ) remaining unreacted at time $t$ will be

$$
\phi\left(k_{i}, t\right)=\phi\left(k_{i}, 0\right) \exp \left[-k_{i} t\right]
$$

It is possible to write similar equations for each of $\mathcal{N}$ species. The weight of total unreacted solid at time $t$ will be

$$
\begin{aligned}
W(t) & =\sum_{i=1}^{N} \phi\left(k_{i} t\right) \\
& =\sum_{i=1}^{N} \phi\left(k_{i}, 0\right) \exp \left[-k_{i} t\right]
\end{aligned}
$$

Because of certain inherent problems associated with discretized distribution mode $\mathrm{e}^{16)}$ it is desirable to use continuous mode of distribution. The overall reaction rate of each single entity contained in the solid can be characterised by a rate constant $0 \leq k \leq k_{\max }$ and those entities whose constants lie in the range $k$ 
to $k+d k$ represent a single species. The distribution of rate constants of species present in the solid at time $t=0$ would be given by the distribution function $\phi(k, 0)$

$$
\int_{0}^{k_{\max }} \phi(k, 0) d k=W_{0}
$$

If the initial mass of the solid is normalized to unit mass, $\phi(k, 0)$ will be the true frequency function and $\phi(k, 0) d k$ will give the fraction of solid having the reaction rate constant between $k$ and $k+d k$. Similarly, if $\phi(k, l)$ is the rate constant distribution function at time $t, \phi(k, t) d k$ will be the fraction of solid (with respect to total unreacted solid at time $t$ ) having the reaction rate constant between $k$ and $k+d k$ at time $t$. Since the fraction of unreacted solid with all rate constants at time $t$ is $W(t)$, the fraction of species (with respect to initial weight of the solid) whose rate constant is in the range $k$ and $k+d k$ is $W(t) \psi(k, t) d k$. Hence,

$$
W(t)=\int_{0}^{k_{\max }} W(t) \psi(k, t) d k
$$

When the reaction is started, more reactive solid species are consumed at a faster rate than the less reactive ones, until all the solid reactant has been consumed. The limiting value of $\phi(k, t)$ would be an impulse at $k=0$. Fig. 2 indicates schematic variation of $\phi(k, t)$ with time. Fig. 3 is schematic three dimensional variation of $W(t) \phi(k, t)$ with rate constant $k$ and time $t$.

Assuming that the rate of reaction of each species is given by first order rate equation

$$
\underline{d}\left[\frac{W(t) \phi(k, t) d k]}{d t}=-k[W(t) \phi(k, t) d k]\right.
$$

Eq. (8) can be written as

$$
\frac{d[W(t) \psi(k, t) d k]}{d t}=-k[W(t) \psi(k, t)]
$$

Integrating from $t=0$ to $t=t$ gives, using $W(0)=1$,

$$
W(t) \phi(k, t)=\phi(k, 0) \exp [-k t]
$$

Combining Eq. (10) with Eq. (7) one gets

$$
W(t)=\int_{0}^{k_{\max }} \phi(k, 0) \exp [-k t] d k
$$

Eq. (11) was encountered earlier in discrete form as Eq. (5). These equations clearly suggest that although the rate constant distribution function itself varies with time, it is possible to analyse the kinetics of reaction (to predict the weight fraction of unreacted solid) from the knowledge of the initial rate constant distribution function, $\phi(k, 0)$, alone.

Differentiation of Eq. (11) with respect to time gives

$$
\frac{d W(t)}{d t}=-\int_{0}^{k_{\max }} k \psi(k, 0) \exp [-k t] d k
$$

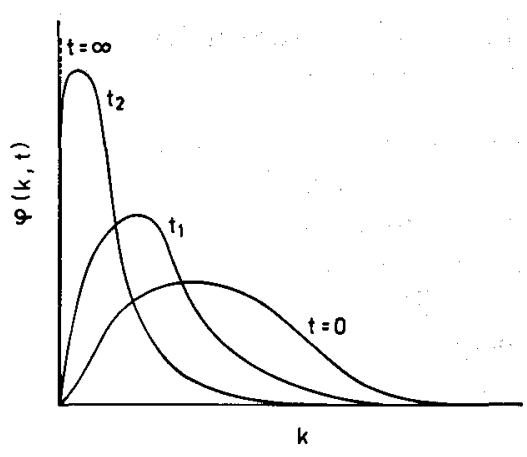

Fig. 2. Schematic variation of $\phi(k, t)$ with time.

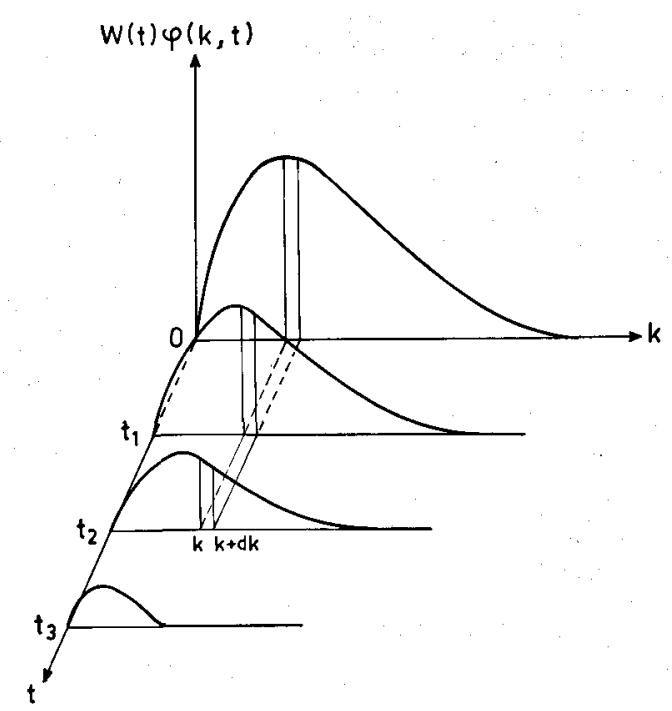

Fig. 3. Schematic three dimensional variation of $W(t) \psi(k$, t) with rate constant and time.

Substituting Eq. (10) in Eq. (12) one gets

$$
\begin{aligned}
\frac{d W(t)}{d t} & =-W(t) \int_{0}^{k_{\max }} k \psi(k, t) d k \\
& =-k_{\mathrm{av}} W(t) \quad \ldots \ldots \ldots \ldots \ldots \ldots
\end{aligned}
$$

where

$$
k_{\mathrm{av}}=\int_{0}^{k_{\max }} k \psi(k, t) d k
$$

Combining Eqs. (10), (11) and (14)

$$
k_{\mathrm{av}}=\frac{\int_{0}^{k_{\max }} k \psi(k, 0) \exp [-k t] d k}{\int_{0}^{k_{\max }} \phi(k, 0) \exp [-k t] d k}
$$

Eq. (13) is, thus, the equation for the average reaction rate of all species at time $t$. It should, however, be recognised that it is a pseudo first order kinetics relationship in $W(l)$ since the average rate constant $k_{\mathrm{av}}$ is a function of time. This explains why in many cases the semilogarithmic plot of weight fraction of unreacted solid vs, time begins to show deviation from linear behaviour after certain degree of conversion. It terms of kinetic steps this deviation is attributed to change in rate controlling step viz. from chemical reaction control to mass transfer control. 


\section{Estimation of Reaction Rate Constant Dis- tribution Function}

From the discussion in the previous section in general, and Eq. (11) in particular, it is obvious that the problem of analysing the first order reaction kinetics in the case of gas-solid reactions is basically the problem of finding the initial reaction rate constant distribution function, i.e., for a given experimental data of $W(t)$ find $\psi(k, 0)$. Effects of operating variables, e.g., temperature, particle size, porosity, etc. on the reaction kinetics can be studied systematically provided an efficient and reliable procedure for computation of rate constant distribution from experimental data is available.

One possible method of finding the rate constant distribution involves assuming a flexible and integrable form for $\psi(k, 0)$ and determining parameters contained in it by using experimental data. The main drawback of such an approach, however, is that the assumed functional form may not be the right one and, hence, lead to erroneous results. This point is illustrated below.

In the analysis on continuum of reaction by Aris, ${ }^{12}$ the rate constant distribution was assumed to be a gamma distribution, i.e.

$$
\phi(k, 0)=\frac{1}{p \Gamma(q)}\left(\frac{k}{p}\right)^{q-1} \exp [-k / p]
$$

where $p$ and $q$ are the two unknown parameters of the distribution and $\Gamma(q)$ is the gamma function. If $k_{\mathrm{av}}$ is the average of reaction rate constants for all species at time $t$, it can be shown that

$$
k_{\mathrm{av}}=\frac{q_{p}}{1+p t}
$$

The $k_{\mathrm{av}}$ values corresponding to $11 \%$ porosity data in Fig. 1 were evaluated at different times. A plot of $k_{\mathrm{av}}^{-1} v s$. time is shown in Fig. 4. The values of $p$ and $q$ were obtained from this plot and found to be -0.011 and -1.89 , respectively. Since the gamma function is not defined for negative values of $q$, it is evident that $\psi(k, 0)$ is not a gamma distribution for

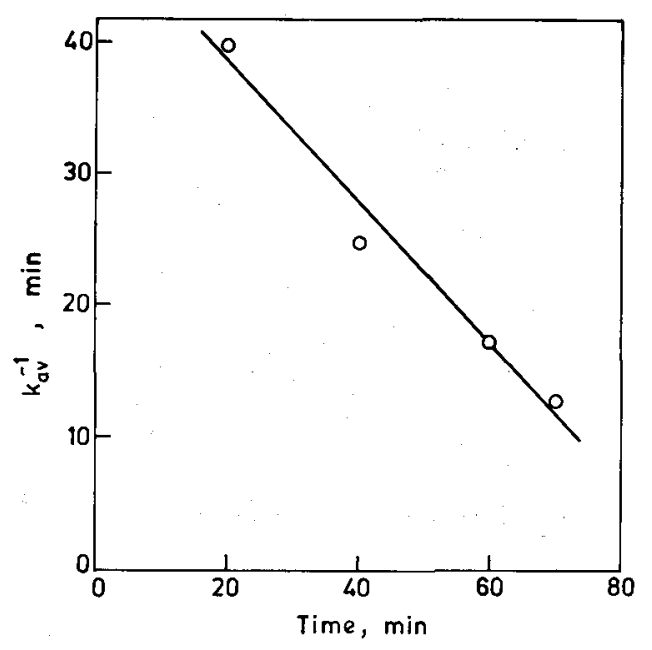

Fig. 4. Plot of $k_{\mathrm{av}}^{-1} v s$. time. the set of data under consideration.

\subsection{Generalized Approach}

A potentially more powerful method of dealing with this problem of computing rate constant distribution is to allow the upper limit of Eq. (11) to go to infinity, i.e.

$$
W(t)=\int_{0}^{\infty} \psi(k, 0) \exp [-k t] d k
$$

It may be recognized here that $W(t)$ in the above equation is the Laplace transform of $\phi(k, 0)$ and, therefore, $\phi(k, 0)$ is inverse of the Lapalce transform. Thus, the problem of determining the reaction rate constant distribution is reduced to finding inverse of Laplace transform using experimental data for $W(t) \cdot{ }^{12)}$ Unfortunately, numerical inversion of the Laplace transform is not an easy task and success is not always assured, especially, in the presence of experimental errors. This is because the Laplace inverse is a very unstable operator which implies that arbitrarily small variations in $W(t)$ may cause large changes in $\psi(k, 0)$. Problems associated with experimental noise in the data have been highlighted by Bcllman et al. ${ }^{17}$ and Mehrotra and Kapur. ${ }^{18)}$ It is perhaps because of the problems associated with the inversion of Laplace transform using noisy experimental data that this technique has not been, hitherto, used in analysis of reaction kinetics.

Mehrotra and Kapur ${ }^{18)}$ developed a numerical method for inversion of the Laplace transform using experimental data. This technique has been used successfully for finding the froth flotation rate constant distribution, ${ }^{19)}$ and energy site distribution for adsorption of gases on solids. ${ }^{20}$ A brief summary of this tcchnique, which is based on discretization of the integral using Lobatto quadrature, and optimization of the resulting constrained variables, is presented below.

It is established that success is more readily assured if the inversion is carried out in the cumulative distribution mode rather than in the form of frequency function. The normalized cumulative distribution function $\phi(k, 0)$ may be defined as

$$
\phi(k, 0)=\int_{k}^{\infty} \phi(k, 0) d k
$$

where

$$
\phi(0,0)=\int_{0}^{\infty} \phi(k, 0) d k=1
$$

and

$$
\psi(k, 0)=-\frac{\partial \phi(k, 0)}{\partial k}
$$

Thus, $\phi(k, 0) d k$ would mean the fraction of material having the reaction rate constant greater than $k$.

Substitution of Eq. (20) in Eq. (18), followed by integration by parts results in

$$
\bar{W}(t)=\int_{0}^{\infty} \phi(k, 0) \exp [-k t] d k
$$


where

$$
\bar{W}(t)=\frac{1-W(t)}{t}
$$

Let

$$
x=2 \exp [-k]-1
$$

Substituting Eq. (24) in Eq. (22)

$$
\bar{W}(t)=\frac{1}{2^{t}} \int_{-1}^{1} \phi\left(\ln \left[\frac{2}{x+1}\right], 0\right)[x+1]^{t-1} d x
$$

Discretizing the integral in the above equation using $n$ point Lobatto quadrature

$$
\bar{W}(t)=\frac{1}{2^{t}}\left[2 \frac{F(1)+F(-1)}{n(n-1)}+\sum_{i=2}^{n-1} H_{i} F\left(x_{i}\right)\right]
$$

where $H_{i}$ and $x_{i}$ are, respectively, weights and abscissas of the Lobatto quadrature formula, ${ }^{21)}$ and

$$
F\left(x_{i}\right)=L\left(x_{i}\right)\left[x_{i}+1\right]^{t-1}
$$

where

$$
L\left(x_{i}\right)=\phi\left(\ln \left[\frac{2}{x_{i}+1}\right], 0\right)
$$

When

$$
k=0, x_{n}=1
$$

and

$$
k=\infty, x_{1}=-1
$$

Moreover,

$$
F(1)=2^{t-1}
$$

and

$$
F(-1)=0
$$

Combining Eqs. (31) and (32) with Eq. (26)

$$
\bar{W}(t)=\frac{1}{n(n-1)}+\frac{1}{2^{t}} \sum_{i=2}^{n-1} H_{i} L\left(x_{i}\right)\left[x_{i}+1\right]^{t-1}
$$

Eq. (33) can be solved for $L\left(x_{i}\right)$, and hence for $\phi(k, 0)$ since $x_{i}$ and $k_{i}$ are related by Eq. (24), using some appropriate optimization technique. A least square objective function of the following form was used:

$$
\begin{aligned}
E= & \sum_{j=1}^{m}\left\{\bar{W}(t)-\frac{1}{n(n-1)}\right. \\
& \left.-\frac{1}{2 t_{j}} \sum_{i=2}^{n-1} H_{i} L\left(x_{i}\right)\left[x_{i}+1\right]^{t_{j}-1}\right\}^{2}
\end{aligned}
$$

where $m$ is the number of data point, $\bar{W}\left(t_{j}\right), j=1,2$, $\ldots, m$.

The unknown variables of the objective function in Eq. (34) are subjected to a number of constraints which arise from the following propertics of the cumulative distribution function:

$$
\phi\left(k_{i}, 0\right) \geq 0,0 \leq k_{i} \leq \infty
$$

(ii) $\phi\left(k_{i}, 0\right)$ is a monotonically non-increasing function of $k_{i}$, i.e.,

$$
1 \geq \phi\left(k_{i} 0\right) \geq \ldots \phi\left(k_{m}, 0\right) \ldots \geq 0
$$

$$
\text { when } \begin{aligned}
& 0 \leq k_{2} \ldots \leq k_{m} \ldots \leq \infty \quad \ldots \ldots \ldots \ldots \\
& \phi(0,0)=1 \text { and } \phi(\infty, 0)=0
\end{aligned}
$$

The Lobatto quadrature is exact at the two extreme points. Hence, the last constraint is automatically satisfied. The second constraint drastically narrows down the region of search to that within the confine of two neighbouring points and prevents wild oscillations. This feature can be readily incorporated in many optimization algorithms such as Univariate Search, ${ }^{22}$ Random Search, ${ }^{23,24)}$ etc.

The 8-point Lobatto quadrature formula was employed to approximate the integral in Eq. (25). In some instances it did not cover adequately the abscissa range of interest specially when function $\phi(k, 0)$ varied sharply over a narrow interval of $k_{i}$. This difficulty could, however, be easily resolved by incorporating an adjustable scaling factor in Eq. (22) as described elsewhere. ${ }^{18)}$ By properly adjusting the value of the scaling factor more points could be generated in the region of interest.

\section{Evaluation of the Method}

The validity of the numerical method for inversion of the Laplace transform has been tested rather rigorously by Mehrotra and Kapur ${ }^{18)}$ using the synthetic data generated from widely different distribution functions. They convincingly showed that the numerical method could always give back the distribution function that was originally used to generate the data to which noise was added randomly to simulate it to experimental data.

In the present study the rate constant distribution functions in the cumulative form, $\phi(k, 0)$, were estimated for the various sets of experimental data taken from literature for the reduction of hematite with hydrogen ${ }^{11,25,26)}$ and for the gasification of carbon/ coke with carbon dioxide ${ }^{10)}$ and unpublished data by the authors. Numerically estimated cumulative rate constant distribution functions are shown in Figs. 5 to 10. To establish that these distribution functions truly represent the systems under consideration, fractions of unreacted reactant materials, as a function of time, were recalculated using Eqs. (23) and (25). For a few sets of data recalculated values of $W(t)$ along with those experimentally measured as a function of time are shown in Figs. 11 to 14 . As can be seen, theoretically predicted values of weight fractions of unreacted materials are in reasonable agreement with those experimentally measured. (For the other sets of data also the agreement was as good as in these figures.)

Another strong evidence in support of this approach comes from the following fact. The plot of $\ln (W(l) \mid$ $\left.W_{0}\right)$ vs. time for the first set of data in Fig. 1 is reasonably close to a straight line. This suggests that the reaction rate constant for the entire material is the same and that its value $\left(k_{c}\right)$ can be obtained from the slope of the straight line. For the single constant value of the rate constant, the frequency function, $\phi(k, 0)$, will be an impulse and the cumulative 
ISIJ International, Vol. 29 (1989), No. 2

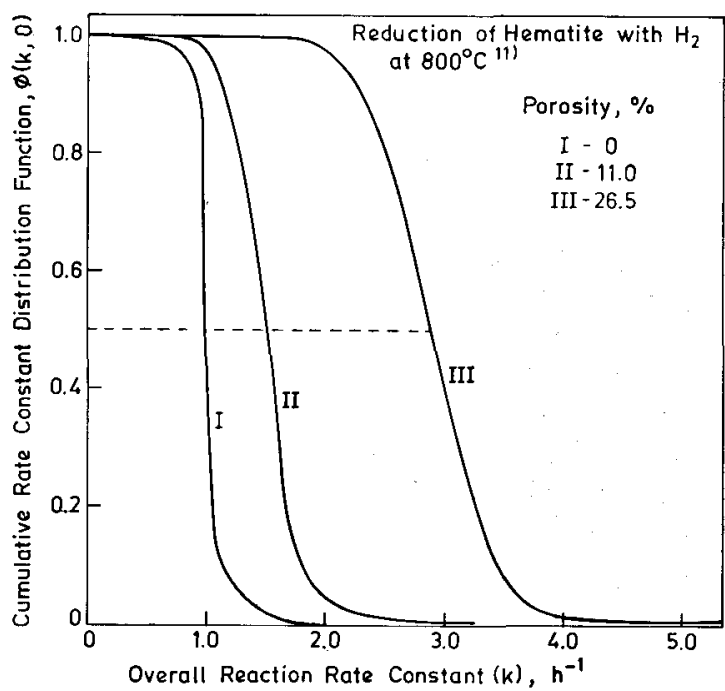

Fig. 5. Cumulative reaction rate constant distribution for reduction of hematite with hydrogen at three different temperatures.

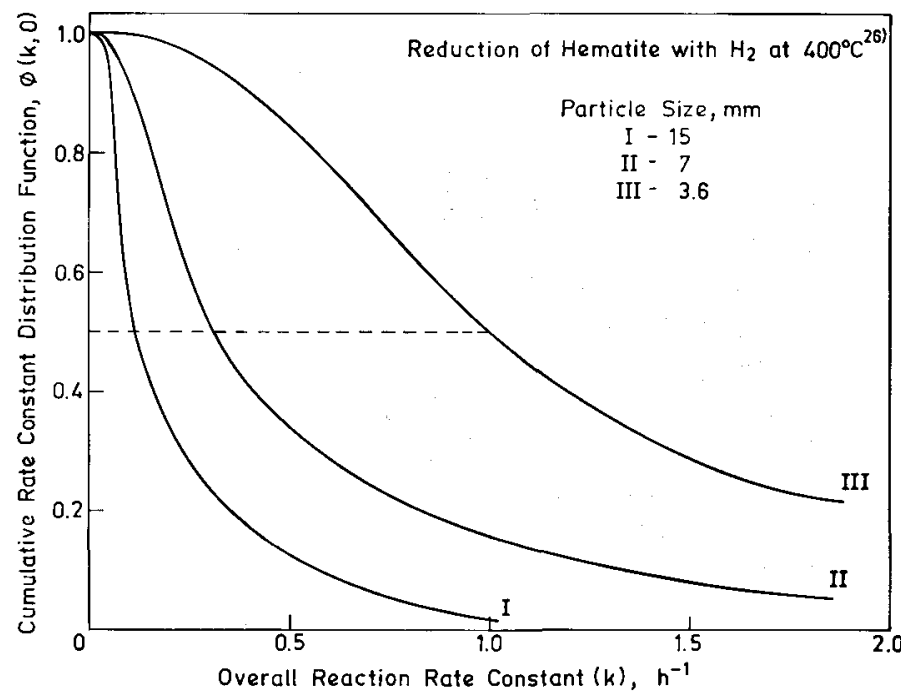

Fig. 7. Cumulative reaction rate constant distribution for reduction of hematite with hydrogen at $400^{\circ} \mathrm{C}$ for pellets of three different porosities.

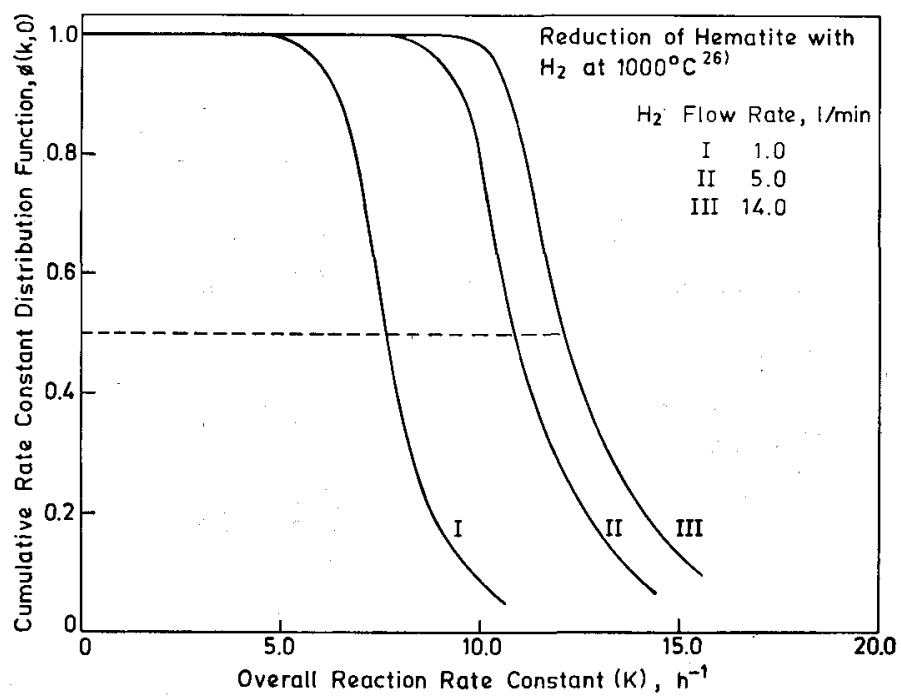

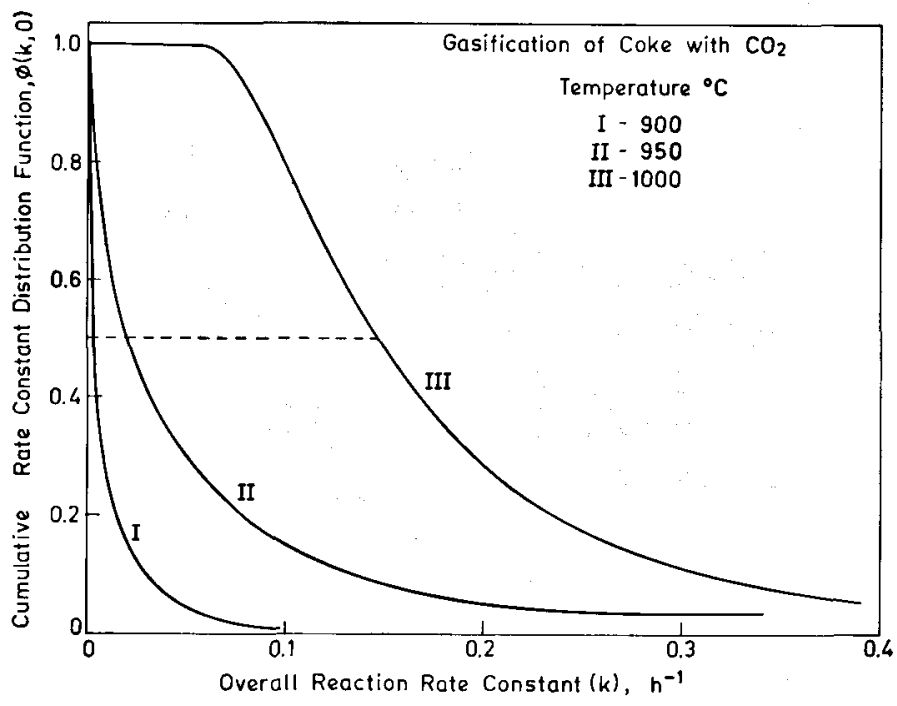

Fig. 6. Cumulative reaction rate constant distribution for gasification of coke with carbon dioxide at three different temperatures.

(Experimental data used in this figure are the unpublished data by the authors.)

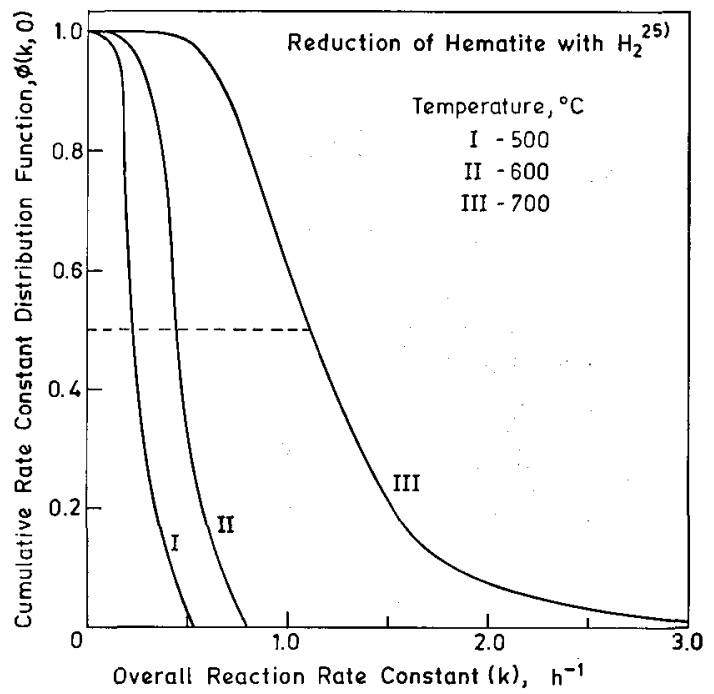

Fig. 8. Cumulative reaction rate constant distribution for reduction of hematite with hydrogen at $800^{\circ} \mathrm{C}$ for pellets of three different porosities.

Fig. 9.

Cumulative reaction rate distribution for reduction of hematite with hydrogen at $1000^{\circ} \mathrm{C}$ at three different flow rates. 


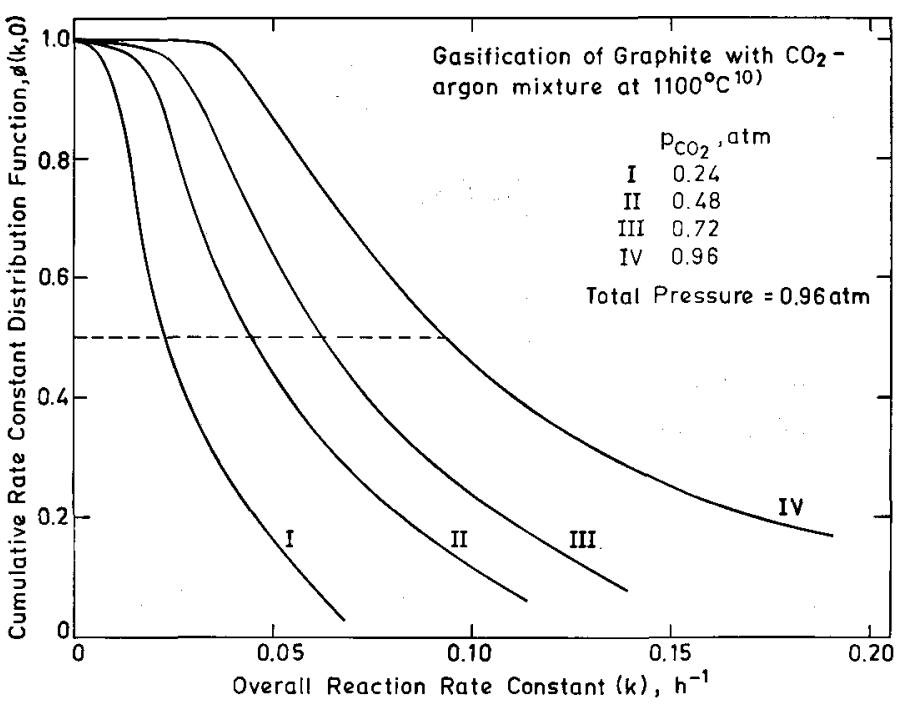

Fig. 10. Cumulative reaction rate distribution for gasification of graphite with $\mathrm{CO}_{2}-\mathrm{Ar}$ mixture at $1100^{\circ} \mathrm{C}$ for four different partial pressures of $\mathrm{CO}_{2}$.

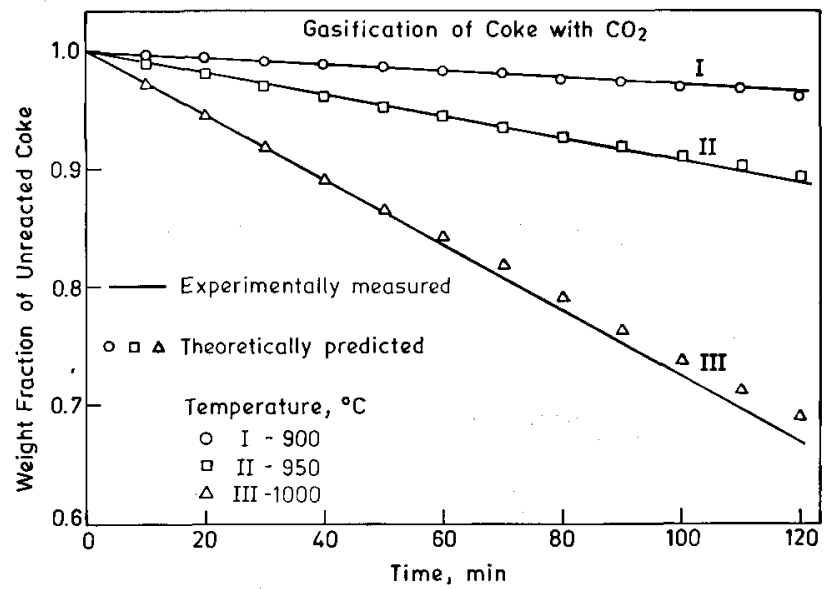

Fig. 12. Experimentally measured and theoretically predicted weight fractions of unreacted coke as a function of time at three different temperatures.

(Experimental data used in this figure are the unpublished data by the authors.)

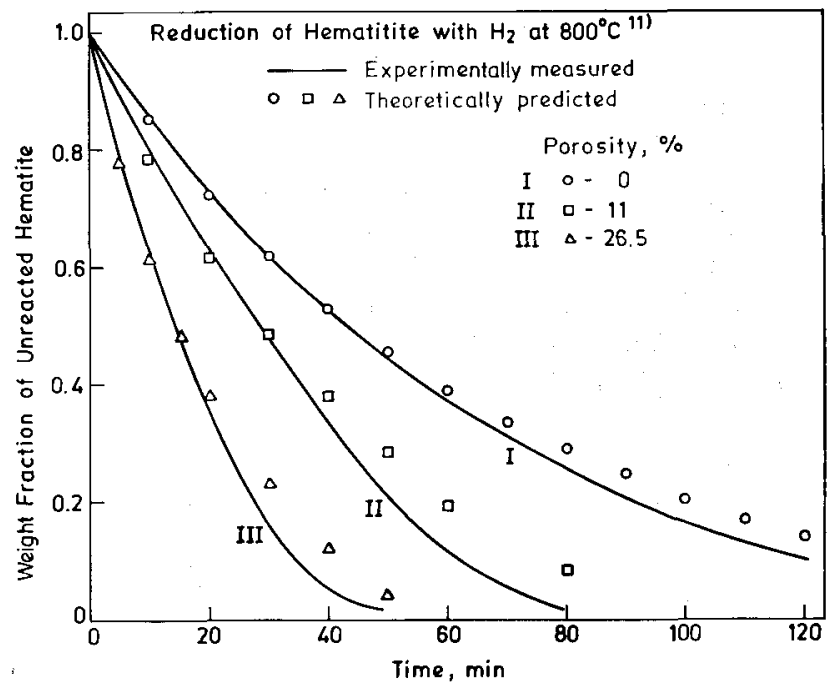

Fig. 14. Experimentally measured and theoretically predicted weight fractions of unreacted hematite as a function of time for pellets of three different porosities.

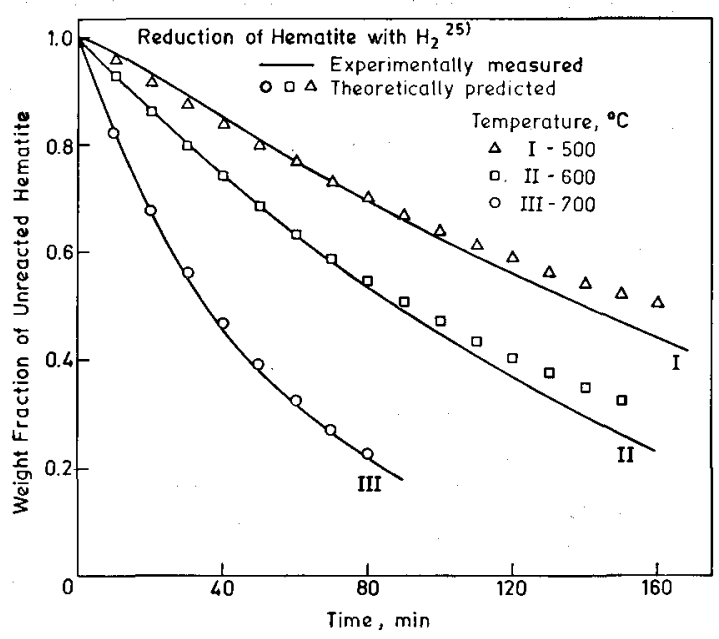

Fig. 11. Experimentally measured and theoretically predicted weight fractions of unreacted hematite as a function of time at three different temperatures.

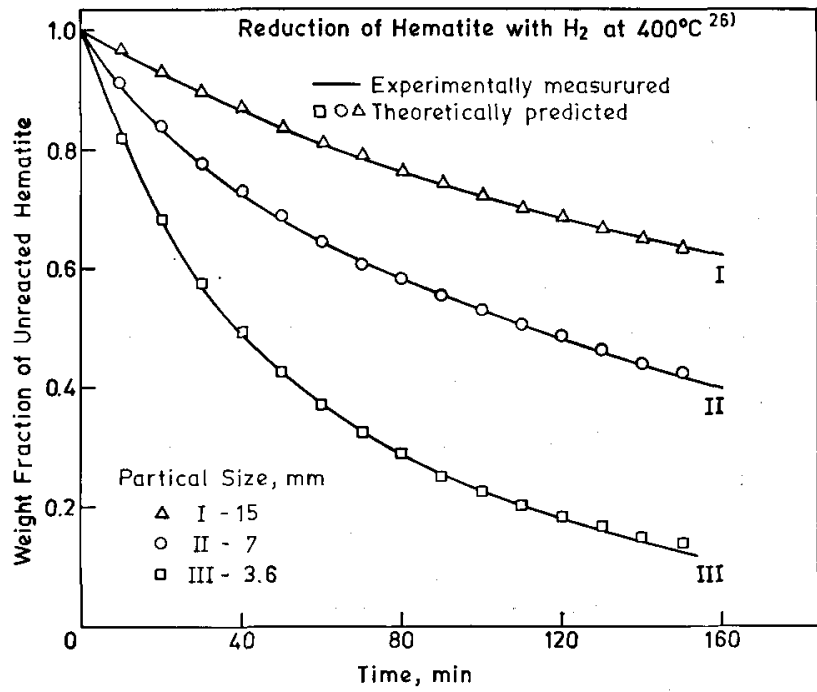

Fig. 13. Experimentally measured and theoretically predicted weight fractions of unreacted hematite as a function of time for pellets of three different sizes.
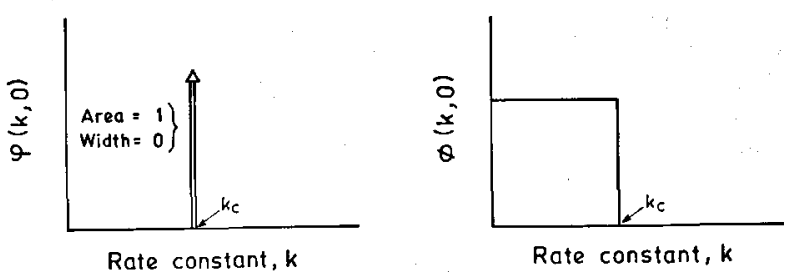

Fig. 15. Schematic representation of the cumulative distribution function when the frequency function is an impulse.

distribution function, $\dot{\phi}(k, 0)$, corresponding to such a frequency function will be a rectangular or uniform distribution as shown in Fig. 15. The value of the rate constant $\left(k_{e}\right)$ obtained from the slope of curve I in Fig. 1 (assuming it to be a straight line) was found to be $1.01 \mathrm{~h}^{-1}$. Close agreement between the numerical values of $k_{c}$ obtained from Figs. 1 and 8, respectively, and the fact that the predicted distribution is, 
indeed, quite close to an uniform distribution clearly establish the validity of this approach.

\section{Application of the Model in Predicting Ef- fects of Process Variables}

Figs. 5 to 10 clearly indicate that, as expected, the rate constant distribution is a strong function of process variables. Effects of some of the common variables viz. temperature, particle size, porosity, gas flow rate and gas composition are qualitatively discussed below.

\subsection{Effect of Temperature}

Figs. 5 and 6 show the effect of temperature on the rate constant distribution-the former figure is for the reduction of hematite with hydrogen whereas the latter is for the gasification of coke with carbon dioxide. It is well known from the conventional reaction kinetics that the chemical reaction rate increases with increasing temperature. In other words, more and more reaction sites become chemically active with increasing temperature. Thus, the increase in intrinsic chemical reactivity of all reaction sites would result in shifting the rate constant distribution towards larger rate constant values. From the comparison of the mean values of distributions in Figs. 5 and 6 (see Table 1) it is evident that the distributions do actually shift towards higher rate constant values with increasing temperaturc.

It is also well established that the rate of the gasification of coke with carbon dioxide is, relatively, a slower process because of lower intrinsic chemical reactivity of coke with carbon dioxide. This fact is clearly revealed in Fig. 6. Comparing the mean

Table 1. Mean values of rate constant distributions for various operating conditions.

\begin{tabular}{|c|c|c|c|c|c|c|}
\hline Fig. & $\begin{array}{l}\text { Tem- } \\
\text { perature } \\
\left({ }^{\circ} \mathrm{C}\right)\end{array}$ & $\begin{array}{c}\text { Particle } \\
\text { size } \\
(\mathrm{mm})\end{array}$ & $\begin{array}{c}\text { Porosity } \\
(\%)\end{array}$ & $\begin{array}{l}\text { Gas } \\
\text { flow } \\
\text { rate } \\
(l / \mathrm{min})\end{array}$ & $\begin{array}{c}\mathrm{CO}_{2} \\
\text { partial } \\
\text { pressure } \\
(\mathrm{atm})\end{array}$ & $\begin{array}{c}k_{\operatorname{mean}} \\
\left(\mathrm{h}^{-1}\right)\end{array}$ \\
\hline \multirow{3}{*}{5} & 500 & & & & & 0.22 \\
\hline & 600 & & & & & 0.45 \\
\hline & 700 & & & & & 1.11 \\
\hline \multirow{3}{*}{6} & 900 & & & & & 0.004 \\
\hline & 950 & & & & & 0.02 \\
\hline & 1000 & & & & & 0.146 \\
\hline \multirow{3}{*}{7} & & 15 & & & & 0.113 \\
\hline & & 7 & & & & 0.312 \\
\hline & & 3.6 & & & & 0.990 \\
\hline \multirow{3}{*}{8} & & & 0 & & & 0.982 \\
\hline & & & 11.0 & & & 1.52 \\
\hline & & & 26.5 & & & 2.88 \\
\hline \multirow{3}{*}{9} & & & & 1.0 & & 7.7 \\
\hline & & & & 5.0 & & 10.8 \\
\hline & & & & 14.0 & & 12.1 \\
\hline \multirow{4}{*}{10} & & & & & 0.24 & 0.022 \\
\hline & & & & & 0.48 & 0.044 \\
\hline & & & & & 0.72 & 0.062 \\
\hline & & & & & 0.96 & 0.094 \\
\hline
\end{tabular}

values of the distributions in Fig. 5 with those in Fig. 6 it becomes obvious that for the coke gasification reaction even at $1000^{\circ} \mathrm{C}$ the mean value of rate constant is only $0.15 \mathrm{~h}^{-1}$ as compared to $0.23 \mathrm{~h}^{-1}$ for the reduction of hematite with hydrogen at temperatures as low as $500^{\circ} \mathrm{C}$.

\subsection{Effect of Particle Size}

It is known from the conventional reaction kinetics that the time necded to react the same fraction of solid reactant for particles of different but unchanging sizes is a function of particle size itself. For example, according to the unreacted core model, for a particle of initial radius $R$ the time $t$ required for a certain fraction of material to react is given by the following. ${ }^{27)}$

$t \propto R^{1.5}$ to 2.0 for film diffusion controlling

$t \propto R^{2} \quad$ for diffusion through the product layer controlling

$t \propto R \quad$ for chemical reaction controlling

In other words, the overall rate of reaction would be inversely proportional to $R^{\prime \prime}$ where the cxponent $y(>1)$ is the weighted sum of the exponents of various kinetic steps.

Effect of particle size on the rate constant distribution is shown in Fig. 7. The shift of the distribution towards larger rate constant values (Table 1) with decreasing particle size is in conformity with the fact that the overall reaction rate is faster for particles of smaller sizes.

\subsection{Effect of Porosity}

Higher porosity of the reactant particle results in lesser resistance to the flow of reactant gas inside the particle. Hence, in the case of a porous particle a larger fraction of the reactant solid would be exposed to the reactant gas as compared to a dense pellet in which the reaction is confined only to the outer surface of the particle in the initial stages of the reaction, and near the interface of unreacted core and ash layer towards the later stages. Thus, in a mass transfer control region, if the entire reactant mass contained in the solid is assumed to have the same intrinsic chemical reactivity, the larger amount of solid being availablc for reaction at a given point of time, in the case of porous particles, would result in higher overall reaction rate. In terms of rate constant distribution, this amounts to saying that the rate constant distibution would shift towards larger values of overall rate constants with increasing porosity of the particle. This, indeed, is happening is evident from Fig. 8 and Table 1.

\subsection{Effects of Gas Flow Rate and Gas Composition}

In terms of reaction mechanism it is known that below a critical gas flow rate the gas film diffusion contributes to overall reaction rate. This is reflected by the fact that below a critical gas flow rate, which depends on particle size, the rate of reaction is a function of gas flow rate but it becomes independent of it above that critical value. In terms of the lumped parameter model the increased gas film resistance at 
low gas flow rates is equivalent to reduction in the overall reaction rate constant. Hence the rate constant distribution is expected to shift towards smaller $k$ values with decreasing gas flow rate. Such a behaviour is indeed exhibited by hematite particles of $15 \mathrm{~mm}$ diameter reduced in hydrogen at three different flow rates (Fig. 9).

A typical plot of rate constant distributions for gasification of graphite in $\mathrm{CO}_{2}-\mathrm{Ar}$ mixture having different partial pressures of $\mathrm{CO}_{2}$ is shown in Fig. 10 . Increased partial pressure (concentration) of the reactant gaseous species is cxpected to increase the rate of reaction implying that, according to our model, the rate constant distribution should shift towards larger $k$ values.

\section{Discussion}

It is recognized that, truly speaking, Eq. (1) is valid for a homogeneous chemical reaction only. Thus, the underlying assumption in the present simulation model is that a heterogeneous gas-solid reaction can be represented by a psuedo first order reaction the rate expression for which is the same as for a homogeneous reaction but with distributed rate constant. The justification for this assumption is partly derived from the following observations:

1) some heterogeneous gas-solid reactions (involving fine particle or porous solid aggregates) in which chemical reaction is rate controlling are represented by Eq. (1), ${ }^{10}$ )

2) even those mass transfer controlled reactions in which the internal pore structure does not change with time are often described by Eq. (1), ${ }^{8)}$

3) the mixture of solid reactants which undergo parallel first order irreversible reactions are lumped using the concept of distribution of species and their rate constants using first order rate equation. ${ }^{12,13,28)}$

It may be reiterated that the rate constant as defined in the present model is a lumped parameter and should be considered only as an overall or apparent rate constant which represents the combined effects of all chemical as well as physical phenomena taking place. Thus, the rate constant distribution is a function of operating variables like particle size, its geometry and morphology, reaction tcmperature, reactor design, gas flow rate, chemical reactivities and physical properties of the reactants, etc. An explicit expression of $\phi(k, 0)$ as a function of these operating and physical parameters is not possible at present.

It may be pointed out that the extension of upper limit of $k$ from $k_{\text {max }}$ to infinity in Eq. (18) is somewhat unrealistic and is used only as a mathematical convenience. In some cases this extension may lead to slight drifting of the distribution towards larger $k$ values. Attempt is now being made to develop a new numerical technique based on non-linear optimization which should lead to estimation of not only the rate constant distribution but also the $k_{\max }$.

It should be recognized that although in the analysis described above only the initial rate constant dis- tribution is required, the rate constant distribution itself is a function of time. Once the initial distribution is known it is possible to predict rate constant distribution at any given point of time using Eq. (10). However, since the rate constant distribution can only be determined using the experimental data, it is necessary to perform a new experiment as and when the reaction conditions are changed. Further, at present, this approach is applicable to a first order reaction only.

One of the main advantages of the present approach is that the kinetic analysis is independent of reaction mechanism. In that sense, it is more general than the conventional approach in which the analysis is based on certain assumed reaction mechanism. From the controversies that exist in literature regarding the reaction mechanisms of even some of the simplest gas-solid reactions it becomes evident that the process of identification of rate controlling steps may indeed be quite cumbersome. Even if in some cases rate controlling steps are identified, quantification of the values of parameters (like the diffusion coefficients, tortuosity factor, equilibrium parameters, etc.) employed in these computations is inevitable. Hence till the time these problems are resolved, any approach like the one developed in this paper would have its own utility. The application of this approach to qualitatively predict the effects of process parameters on reaction kinetics has already been described above. An attempt is now being made to quantify these effects.

\section{Summary and Conclusions}

It has been shown that without going into the details of reaction mechanisms it is possible to analyse the overall reaction kinetics of first order gas-solid reactions by approximating the heterogeneous reaction to a homogeneous reaction with distributed reaction rate constant. The solid reactant is considered as consisting of reaction species having different overall reaction rate constants and thus a rate constant distribution is associated with the solid. The problem of analysing reaction kinetics is reduced to finding the rate constant distribution which, in continuous mode, is shown to be equivalent to the problem of finding the inverse of the Laplace transform using the experimental kinetic data. The numerical technique used for this purpose involves discretization of the integral contained in the kinetic equation by 8-point Lobatto quadrature and then carrying out constrained nonlinear optimization. The rate constant distributions were evaluated for various sets of data, taken from literature, for the reduction of hematite with hydrogen and gasification of carbon/coke with carbon dioxide. Effects of temperature, particle size, porosity, gas flow rate and gas composition on the rate constant distributions have been qualitatively discussed.

\section{Nomenclature}

E: Objective function defined by Eq. (34) 
$F\left(x_{i}\right):$ Function defined by Eq. (27)

$H_{i}$ : Weights of 8-point Lobatto quadrature

$k$ : Reaction rate constant $\left(\mathrm{h}^{-1}\right)$

$k_{\mathrm{av}}$ : Average value of reaction rate constants for all species at time $t$

$k_{c}$ : Value of the reaction rate constant when the frequency function is an impulse

$k_{i}$ : Reaction rate constant of species $i$

$k_{\max }$ : Maximum value of reaction rate constant $k$

$k_{\text {mean }}$ : Mean value of the cumulative distribution function

$L\left(x_{i}\right):$ Function defined by Eq. (28)

$m$ : Number of data points used in computations of distribution function

$n$ : Order of Lobatto quadrature used for discretizing the integral in the rate equation

$\mathcal{N}$ : Number of species contained in the reactant solid

$p$ : Parameter of gamma distribution defined by Eq. (16)

$q$ : Parameter of gamma distribution defined by Eq. (16)

$R$ : Radius of the reacting particle

$t$ : Time (min)

$W_{0}$ : Initial mass of reactant solid (Normalized to unit mass)

$W(t)$ : Weight fraction of unreacted solid at time $t$

$\bar{W}(l):$ As defined by Eq. (23)

$x$ : Variable defined by Eq. (24)

$x_{i}:$ Abscissas of 8-point Lobatto quadrature

$Y$ : Exponent on $R$ in the relationship defining reaction rate as a function of particle size

$\phi(k, 0)$ : Normalized cumulative rate constant distribution function at time $t=0$

$\phi(k, 0)$ : Continuous rate constant frequency function at time $t=0$

$\phi(k, t)$ : Continuous rate constant frequency function at time $t=t$

$\phi\left(k_{i}, 0\right)$ : Discrete rate constant frequency function at time $t=0$

$\psi\left(k_{i}, t\right):$ Discrete rate constant frequency function at time $t=t$

\section{Acknowledgement}

The authors would like to acknowledge that financial support for this research project was provided by the National Science and Engineering Research Council of Canada.

\section{REFERENCES}

1) A. V. Bradshaw: Trans. Inst. Min. Metall, 79C (1970),
281.

2) N.J. Themelis and W. H. Gauvin: Trans. Metall. Soc. AIME, 227 (1963), 290.

3) R. G. Olsson and W. M. McKewan: Trans. Metall. Soc. AIME, 236 (1966), 1518.

4) J. Szekely and H. Y. Sohn: Trans. Inst. Min. Metall., 82 C (1973), 99 .

5) B.B.L. Seth and H. V. Ross: Trans. Metall. Soc. AIME, (1965), 180.

6) R. H. Spitzer, F. S. Manning and W. O. Philbrook: Trans. Metall. Soc. AIME, 236 (1966), 726.

7) W. K. Lu and G. Bitsianes: Trans. Metall. Soc. AIME, 236 (1966), 531 .

8) Y. K. Rao and B. P. Jalan: Metall. Trans., 3 (1972), 2465.

9) R. H. Tien and E. T. Turkdogan: Metall. Trans., 3 (1972), 2039.

10) E. T. Turkdogan, V. Koump, J. V. Vinters and T. F. Perzak: Carbon, 6 (1968), 467.

11) W. M. McKewan: Trans. Metall. Soc. AIME, 212 (1958), 791.

12) R. Aris: Arch. Rat. Mech. Anal., 27 (1968), 356.

13) P. Hutchinson and D. Luss: Chem. Eng. J., 1 (1971), 129.

14) C. C. Harris and A. Chakravarti: Trans. Am. Inst. Min. Eng., 247 (1973), 162.

15) P. C. Kapur and S. P. Mehrotra: Trans. Inst. Min. Metall., 82C (1973), 229.

16) C. Lanczos: Applied Analysis, Prentice Hall, New Jersey, (1961), 1267.

17) R. E. Bellman, R. E. Kalaba and J. E. Lockett: Numerical Inversion of Laplace Transform: Application to Biology, Economics, Engineering and Physics, Elsevier, New York, (1966), 17.

18) P. C. Kapur and S. P. Mehrotra: Chem. Eng. Sci., 29 (1974), 411.

19) S. P. Mehrotra and P. G. Kapur: Powder Technology, 9 (1974), 213.

20) S. P. Mehrotra: Colloids and Surfaces, 3 (1981), 267.

21) Z. Kopal: Numerical Analysis, John Wiley \& Sons, New York, (1961), 573.

22) S. G. Beveridge and R. S. Schechter: OptimizationTheory and Practice, McGraw-Hill, New York, (1970).

23) E. S. Lee: Quasilinearization and Invariant ImbeddingMathematics in Science and Engineering, Academic Press, New York, 41 (1968), 95.

24) R. Luus and T.H.I. Jaakola: AIChE J., 19 (1973), 760

25) P. K. Strangeway and H. U. Ross: " Kinetics of Magnetite Reduction ", Paper Presented at the Annual AIME Meeting, Los Angles, 1967, AIME.

26) E. T. Turkdogan and J. V. Vinters: Metall. Trans., 2 (1971), 3175

27) O. Levenspiel: Chemical Engineering Kinetics, 2nd Ed., John Wiley \& Sons, New York, (1972), 375.

28) R. Aris and G. R. Gavalas: Phil. Trans. Roy. Soc., A260 (1966), 351 . 